\title{
ON FUNCTIONAL DEPENDENCIES AND MULTIVALUED DEPENDENCIES IN FUZZY RELATIONAL DATABASES
}

\author{
HO THUAN, TRAN THIEN THANH
}

\begin{abstract}
In this paper, we present a new definition of fuzzy functional depencency and fuzzy multivalued dependency based on similarity in fuzzy relational database, for which thresholds are defined for each attributes of relation scheme. The soundness and completeness of inference rules, similar to Armstrong's axioms are proved.
\end{abstract}

Tóm tăt. Trong bài báo này chúng tôi trình bày định nghĩa cho phụ thuộc hàm và phụ thuộc đa trị mò̀ trên mô hình co* sơ dũ liệu mò dựa trên quan hệ tưong tự với ngướng tương tự xác định riêng cho mỗi thuộc tính. Tính xác đáng và đầy đư cửa hệ tiên đề tưong tự hệ tiên đề Armstrong cũng được chúng minh.

\section{INTRODUCTION}

Relational databases have been studied since Codd's. Such databases can only deal with welldefined and unambiguous data. But in the real world there exist data which cannot be well-defined in a certain clear sense and under a certain crisp form (often called fuzzy data). The databases for the above mentioned data have been investigated by different authors (see [7]). The fuzzy database models are an extension of the classical relational model. It is based on the fuzzy set theory invented by Zadeh to capture the imprecise parts of the real world.

In genegal, two approaches have been proposed for the introduction of fuzziness in the relational model. The first one uses the principle of replacing the ordinary equivalence among domain values by measures of nearness such as similarity relationships, proximity relationship, and distinguishability function (see [8]). The second major effort has involved a variety of approaches that directly use possibility distributions for attribute value (see [5]). There have also been some mixed models combining these approaches [12].

This paper takes the similarity-based fuzzy relational databases as the reference model in our study presented here.

The data dependencies are the most important topics in theory of relational databases. Several authors have proposed extended dependencies in fuzzy relational database models. In $[1,2,4,6,10,12]$ have been given definitions of fuzzy functional dependencies and fuzzy multivalued dependencies in fuzzy relational data models. These dependencies are extension of dependencies of classical relational model. In this article, we give the definitions of fuzzy functional dependency (abbr. $(\alpha, \beta)$-ffd) and fuzzy multivalued dependency (abbr. $(\alpha, \beta)$-fmvd). These dependencies are extention of dependencies in classical model and more general than definitions of Rauj, Mazumdar, etc. We also show that the inference rules of $(\alpha, \beta)$-ffd, $(\alpha, \beta)$-fmvd, which are similar to Armstrong' axioms for classical relational databases, are sound and complete.

This paper is organized as follows. Section 2 present some basic definitions of the similaritybased relational databases. In Section 3 and Section 4, we introduce an extension of functional and multivalued dependencies; Armstrong's axioms for fuzzy functional and multivalued dependencies are presented; the soundness and completeness are proved. Section 5 concludes this paper with some perspectives of the present work.

\section{SIMILARITY-BASED FUZZY RELATIONAL DATABASES}

The similarity-based fuzzy relational database model is a generalization of the original relational model. It is allowed an attribute value to be a subset of the associated domain. Domains for this model are either discrete scalars or discrete numbers drawn from either a finite or infinite set. The 
equivalence relation over the domain is replaced by a fuzzy similarity relation to identify similar tuples exceeding a given threshold of similarity.

Definition 2.1. A similarity relation is a mapping $s: D \times D \rightarrow[0,1]$ such that for $x, y, z \in D$,

$$
\begin{aligned}
& s(x, x)=1 \text { (reflexivity), } \\
& s(x, y)=s(y, x) \text { (symmetry), } \\
& s(x, z) \geq \max _{y \in D}\{\min [s(x, y), s(y, z)]\} \text { (max-min transitivity). }
\end{aligned}
$$

Definition 2.2. A fuzzy relation scheme is a triple $S=(R, \vec{s}, \vec{\alpha})$, where $R=\left\{A_{1}, A_{2}, \ldots, A_{n}\right\}$ is a set of attributes, $\vec{s}=\left(s_{1}, s_{2}, \ldots, s_{n}\right)$ is a set of associated similarity relations, $\vec{\alpha}=\left(\alpha_{1}, \alpha_{2}, \ldots, \alpha_{n}\right)$ is a set of associated thresholds $\left(\alpha_{i} \in[0,1], 1 \leq i \leq n\right)$.

Definition 2.3. A fuzzy relation instance $r$ on scheme $S=(R, \vec{s}, \vec{\alpha})$ is a subset of the cross product $P\left(D_{1}\right) \times P\left(D_{2}\right) \times \cdots \times P\left(D_{n}\right)$, where $D_{i}=\operatorname{dom}\left(A_{i}\right)$, and $P\left(D_{i}\right)=2^{D_{i}}-\emptyset$.

Let $X, Y$ be sets of attributes in $R, X=\left(A_{h}\right)_{h \in I}, I \subseteq\{1, \ldots, n\}$.

$\alpha_{X}$ denotes the vector of thresholds for a set of attributes $X$, i.e. $\alpha_{X}=\left(\alpha_{h}\right)_{h \in I}$.

$\alpha_{X Y}$ denotes the vector of thresholds for a set of attributes $X \cup Y$ ( $X Y$ for short).

In order to approximate equality between tuples of fuzzy relation, a fuzzy measure, a similarity relation $\tau$ is defined as follows.

Definition 2.4. Let $r$ be a fuzzy relation instance on scheme $S=(R, \vec{s}, \vec{\alpha}), t_{1}$ and $t_{2}$ are two tuples in $r$. The similarity measure of two tuples $t_{1}$ and $t_{2}$ on attribute $A_{k}$ in $R$ denoted by $\tau\left(t_{1}\left[A_{k}\right], t_{2}\left[A_{k}\right]\right)$ is given as

$$
\tau\left(t_{1}\left[A_{k}\right], t_{2}\left[A_{k}\right]\right)=\min _{x \in d_{k}^{1}, y \in d_{k}^{2}}\left\{s_{k}(x, y)\right\}
$$

where $t_{1}=\left(d_{1}^{1}, d_{2}^{1}, \ldots, d_{n}^{1}\right), t_{2}=\left(d_{1}^{2}, d_{2}^{2}, \ldots, d_{n}^{2}\right)$.

If $\tau\left(t_{1}\left[A_{k}\right], t_{2}\left[A_{k}\right]\right) \geq \alpha_{k}$ then tuples $t_{1}$ and $t_{2}$ are said to be similar on $A_{k}$ with threshold $\alpha_{k}$.

Definition 2.5. Let $r$ be a fuzzy relation on scheme $S=(R, \vec{s}, \vec{\alpha}), X$ be a subset of $R, t_{1}$ and $t_{2}$ are two tuples in $r$. The similarity measure of two tuples $t_{1}$ and $t_{2}$ on a set of attributes $X$ denoted by $\tau\left(t_{1}[X], t_{2}[X]\right)$ is given as

$$
\tau\left(t_{1}[X], t_{2}[X]\right)=\left(\tau\left(t_{1}\left[A_{i_{1}}\right], t_{2}\left[A_{i_{1}}\right]\right), \tau\left(t_{1}\left[A_{i_{2}}\right], t_{2}\left[A_{i_{2}}\right], \ldots, \tau\left(t_{1}\left[A_{i_{k}}\right], t_{2}\left[A_{i_{k}}\right]\right)\right),\right.
$$

where $X=A_{i_{1}} A_{i_{2}} \ldots A_{i_{k}}$.

If $\tau\left(t_{1}[X], t_{2}[X]\right) \geq \alpha_{X}$ then two tuples $t_{1}$ and $t_{2}$ are said to be similar on $X$ with thresholds $\alpha_{X}$.

\section{FUZZY FUNCTIONAL DEPENDENCY $(\alpha, \beta)$-FFD}

Definition 3.1. Let $r$ be any fuzzy relation instance on scheme $S=(R, \vec{s}, \vec{\alpha}), X$ and $Y$ are subsets of $R$ with associated thresholds $\alpha_{X}, \alpha_{Y}$, respectively. Fuzzy relation instance $r$ is said to satisfy the fuzzy functional dependency $(\alpha, \beta)$-ffd, denoted by $X \underset{\left(\alpha_{X}, \alpha_{Y}\right)}{\sim} Y$ if, for every pair of tuples $t_{1}$ and $t_{2}$ in $r, \tau\left(t_{1}[X], t_{2}[X]\right) \geq \alpha_{X}$ then $\tau\left(t_{1}[Y], t_{2}[Y]\right) \geq \alpha_{Y}$.

Definition 3.2. A scheme $S=(R, \vec{s}, \vec{\alpha})$ is said to satisfy the $(\alpha, \beta)$-ffd $X \underset{\left(\alpha_{X}, \alpha_{Y}\right)}{\sim} Y$, if every relation instance $r$ on $S$ satisfies $X \underset{\left(\alpha_{X}, \alpha_{Y}\right)}{\sim} Y$.

Remark 1. The definition ffd of Raju et al. is a special case of Defifition 3.1. (i.e., if any instance $r$ that satisfies ffd $X \sim \alpha_{0} Y$ then $r$ also satisfies $(\alpha, \beta)$-ffd $\left.X \underset{\left(\alpha_{X}, \alpha_{Y}\right)}{\sim} Y\right)$, where $\alpha_{X}=(\underbrace{\alpha_{0}, \alpha_{0}, \ldots, \alpha_{0}})$ and $\alpha_{Y}=(\underbrace{\alpha_{0}, \alpha_{0}, \ldots, \alpha_{0}}_{|Y| \text { times }})$ with $\alpha_{0}$ is a constant in $\left.[0,1]\right)$.

$|X|$ times

The inference rules for $(\alpha, \beta)$-ffds

FFD1 (Reflexivity): If $Y \subseteq X$ then $X \underset{\left(\alpha_{X}, \alpha_{Y}\right)}{\sim} Y$

FFD2 (Augmentation): If $X \underset{\left(\alpha_{X}, \alpha_{Y}\right)}{\sim \sim} Y$ then $X W \underset{\left(\alpha_{X} \underset{W}{\sim}, \alpha_{Y W}\right)}{\sim} Y W$ 
FFD3 (Transitivity): If $X \underset{\left(\alpha_{X}, \alpha_{Y}\right)}{\sim} Y$, and $Y \underset{\left(\alpha_{Y}, \alpha_{Z}\right)}{\sim} Z$ then $X \underset{\left(\alpha_{X}, \alpha_{Z}\right)}{\sim} Z$

Theorem 3.1. Rules FFD1-FFD3 are sound.

Proof. Let $r$ be a relation instance on scheme $S=(R, \vec{s}, \vec{\alpha})$.

Reflexivity: Suppose that $Y \subseteq X \subseteq R$.

$\forall t_{1}, t_{2} \in r, \tau\left(t_{1}[X], t_{2}[X]\right) \geq \alpha_{X}$. Since $X \subseteq Y$, then $\tau\left(t_{1}[Y], t_{2}[Y]\right) \geq \alpha_{Y}$. Thus $X \underset{\left(\alpha_{X}, \alpha_{Y}\right)}{\longrightarrow} Y$ holds in $r$.

Augmentation: Suppose that $X \underset{\left(\alpha_{X}, \alpha_{Y}\right)}{\sim} Y$ holds in $r, Z \subseteq R$.

$\forall t_{1}, t_{2} \in r, \tau\left(t_{1}[X Z], t_{2}[X Z]\right) \geq \alpha_{X Z}$.

We have $\tau\left(t_{1}[X], t_{2}[X]\right) \geq \alpha_{X}$ and $\tau\left(t_{1}[Z], t_{2}[Z]\right) \geq \alpha_{Z}$.

Since $X \underset{\left(\alpha_{X}, \alpha_{Y}\right)}{\sim} Y$ holds in $r$ then $\tau\left(t_{1}[Y], t_{2}[Y]\right) \geq \alpha_{Y}$.

Combining (1) with (2), we obtain $\tau\left(t_{1}[Y Z], t_{2}[Y Z]\right) \geq \alpha_{Y Z}$.

Hence $X Z \underset{\left(\alpha_{X Z}, \alpha_{Y Z}\right)}{\sim} Y Z$ holds in $r$.

Transitivity: Suppose that $X \underset{\left(\alpha_{X}, \alpha_{Y}\right)}{\sim} Y$, and $Y \underset{\left(\alpha_{Y}, \alpha_{Z}\right)}{\sim} Z$ hold in $r$.

$\forall t_{1}, t_{2} \in r, \tau\left(t_{1}[X], t_{2}[X]\right) \geq \alpha_{X}$.

Since $X \underset{\left(\alpha_{X}, \alpha_{Y}\right)}{\sim} Y$ holds in $r$ then $\tau\left(t_{1}[Y], t_{2}[Y]\right) \geq \alpha_{Y}$.

Since $Y \underset{\left(\alpha_{Y}, \alpha_{Z}\right)}{\sim} Z$ holds in $r$ then $\tau\left(t_{1}[Z], t_{2}[Z]\right) \geq \alpha_{Z}$.

Therefore, $Y \underset{\left(\alpha_{Y}, \alpha_{Z}\right)}{\sim} Z$ holds in $r$.

The following rules are easily obtained from FFD1-FFD3.

FFD4 (Union): $\quad$ If $X \underset{\left(\alpha_{X}, \alpha_{Y}\right)}{\sim} Y$ and $X \underset{\left(\alpha_{X}, \alpha_{Z}\right)}{\sim \sim} Z$ then $X \underset{\left(\alpha_{X}, \alpha_{Y Z}\right)}{\sim} Y Z$

FFD5 (Pseudo-transitivity): If $X \underset{\left(\alpha_{X}, \alpha_{Y}\right)}{\sim} Y$ and $Y W \underset{\left(\alpha_{Y W}, \alpha_{Z}\right)}{\sim} Z$ then $X W \underset{\left(\alpha_{X W}, \alpha_{Z}\right)}{\sim} Z$

FFD6 (Decomposition): If $X \underset{\left(\alpha_{X}, \alpha_{Y}\right)}{\sim} Y$ and $Z \subseteq Y$ then $X \underset{\left(\alpha_{X}, \alpha_{Z}\right)}{\sim} Z$

Theorem 3.2. Rules FFD1-FFD6 are complete on scheme $S=(R, \vec{s}, \vec{\alpha})$ when the following condition holds:

For each $A_{i} \in R$, there exists at least one pair of elements $a_{i}, b_{i} \in \operatorname{dom}\left(A_{i}\right)$ such that $s_{i}\left(a_{i}, b_{i}\right) \leq \alpha_{i}$.

Proof. Let $F$ be a set of $(\alpha, \beta)$-ffds on scheme $S=(R, \vec{s}, \vec{\alpha})$, and suppose that $f=X_{\left(\alpha_{X}, \alpha_{Y}\right)}^{\sim} Y$ does not follow from $F$ by the rules FFD1-FFD6.

Consider the relation instance $r$ on scheme $S$ with two tuples as follows

\begin{tabular}{ccccccc}
\multicolumn{3}{c}{ Attributes of $X^{+}$} & \multicolumn{3}{c}{ Other attributes } \\
\hline$a_{1}$ & $a_{2}$ & $\ldots$ & $a_{k}$ & $a_{k+1}$ & $\ldots$ & $a_{n}$ \\
$a_{1}$ & $a_{2}$ & $\ldots$ & $a_{k}$ & $b_{k+1}$ & $\ldots$ & $b_{n}$
\end{tabular}

It is easily shown that all $(\alpha, \beta)$-ffds in $F$ are satisfied by $r$, and $f$ is not satisfied by $r$.

We conclude that whenever $X \underset{\left(\alpha_{X}, \alpha_{Y}\right)}{\sim} Y$ does not follow from $F$ by the rules FFD1-FFD6 then $F$ does not logically imply $X \underset{\left(\alpha_{X}, \alpha_{Y}\right)}{\sim} Y$. That is, the rules FFD1-FFD6 are complete.

\section{FUZZY MULTIVALUED DEPENDENCY $(\alpha, \beta)$-FMVD}

Definition 4.1. Let $r$ be any fuzzy relation instance on scheme $S=(R, \vec{s}, \vec{\alpha}), X$ and $Y$ are subsets of $R$, with associated thresholds $\alpha_{X}, \alpha_{Y}$, respectively. Relation $r$ is said to satisfy the fuzzy multivalued dependency $(\alpha, \beta)$-fmvd, denoted by $X \underset{\left(\alpha_{X}, \alpha_{Y}\right)}{\sim \longrightarrow} Y$ if, for every two tuples $t_{1}, t_{2}$ in $r, \tau\left(t_{1}[X], t_{2}[X]\right) \geq$ $\alpha_{X}$ then there exists a tuple $t_{3}$ in $r$ such that $\tau\left(t_{1}[X], t_{3}[X]\right) \geq \alpha_{X}, \tau\left(t_{1}[Y], t_{3}[Y]\right) \geq \alpha_{Y}$, and $\tau\left(t_{2}[Z], t_{3}[Z]\right) \geq \alpha_{Z}$, where $Z=R-X Y$. 
Definition 4.2. A scheme $S=(R, \vec{s}, \vec{\alpha})$ is said to satisfy the $(\alpha, \beta)$-fmvd $X \underset{\left(\alpha_{X}, \alpha_{Y}\right)}{\sim} Y$ if every relation instance $r$ on $S$ satisfies $X \underset{\left(\alpha_{X}, \alpha_{Y}\right)}{\sim \longrightarrow} Y$.

Remark 2. The definition fmvd of Mazumdar et al. [1] is a special case of Definition 4.1 (i.e. if relation $r$ holds fmvd $X \sim \alpha_{0} Y$ then $r$ also holds $(\alpha, \beta)$-fmvd $X \underset{\left(\alpha_{X}, \alpha_{Y}\right)}{\sim} Y$, where $\alpha_{X}=(\underbrace{\alpha_{0}, \alpha_{0}, \ldots, \alpha_{0}})$ and $\left.\alpha_{Y}=(\underbrace{\alpha_{0}, \alpha_{0}, \ldots, \alpha_{0}}_{|Y| \text { times }})\right)$.

$|X|$ times

By Defifition 4.1 it is easy to show following remarks.

Remark 3. Relation $r$ satisfies $X \underset{\left(\alpha_{X}, \alpha_{Y}\right)}{\sim} Y$ if, for every two tuples $t_{1}, t_{2}$ in $r, \tau\left(t_{1}[X], t_{2}[X]\right) \geq \alpha_{X}$ then there exists a tuple $t_{3}$ in $r$ such that $\tau\left(t_{2}[X], t_{3}[X]\right) \geq \alpha_{X}, \tau\left(t_{2}[Y], t_{3}[Y]\right) \geq \alpha_{Y}$, and $\tau\left(t_{1}[Z], t_{3}[Z]\right) \geq$ $\alpha_{Z}$, where $Z=R-X Y$.

Remark 4. Relation $r$ satisfies $X \underset{\left(\alpha_{X}, \alpha_{Y}\right)}{\sim} Y$ if, for every two tuples $t_{1}, t_{2}$ in $r, \tau\left(t_{1}[X], t_{2}[X]\right) \geq \alpha_{X}$ then there exists a tuple $t_{3}$ in $r$ such that $\tau\left(t_{1}[X Y], t_{3}[X Y]\right) \geq \alpha_{X Y}$, and $\tau\left(t_{2}[X(R-Y)], t_{3}[X(R-Y)]\right) \geq$ $\alpha_{X(R-Y)}$.

The inference rules for $(\alpha, \beta)$-fmvds.

FMVD1 (Complementation): If $X \underset{\left(\alpha_{X}, \alpha_{Y}\right)}{\sim \longrightarrow} Y$ then $X \underset{\left(\alpha_{X}, \alpha_{Z}\right)}{\sim \cdots} Z$, where $Z=R-X Y$

FMVD2 (Augmentation): If $X \underset{\left(\alpha_{X}, \alpha_{Y}\right)}{\sim} Y, V \subseteq W$ then $X W \underset{\left(\alpha_{X W}, \alpha_{Y V}\right)}{\sim} Y V$

FMVD3 (Transitivity): If $X \underset{\left(\alpha_{X}, \alpha_{Y}\right)}{\sim \longrightarrow} Y$ and $Y \underset{\left(\alpha_{X}, \alpha_{Z}\right)}{\sim \longrightarrow} Z$ then $X \underset{\left(\alpha_{X}, \alpha_{(Z, Y}\right)}{\sim}(Z-Y)$

Theorem 4.1. Rules FMVD1-FMVD3 are sound.

Proof. Let $r$ be a relation instance on scheme $S=(R, \vec{s}, \vec{\alpha})$.

Complementation: Suppose $X \underset{\left(\alpha_{X}, \alpha_{Y}\right)}{\sim} Y$ holds in $r$.

$\forall t_{1}, t_{2} \in r, \tau\left(t_{1}[X], t_{2}[X]\right) \geq \alpha_{X}$.

Since $X \underset{\left(\alpha_{X}, \alpha_{Y}\right)}{\underset{\tau}{\longrightarrow}} Y$ then $\exists t_{3} \in r$, such that

$$
\begin{aligned}
& \tau\left(t_{2}[X], t_{3}[X]\right) \geq \alpha_{X}, \\
& \tau\left(t_{2}[Y], t_{3}[Y]\right) \geq \alpha_{Y}, \\
& \tau\left(t_{1}[Z], t_{3}[Z]\right) \geq \alpha_{Z}, \text { where } Z=R-X Y .
\end{aligned}
$$

Since $W=R-X Z \subseteq Y$, and by (3) then $\tau\left(t_{2}[W], t_{3}[W]\right) \geq \alpha_{W}$.

From (5) and (6), it follows that $X \underset{\left(\alpha_{X}, \alpha_{Z}\right)}{\sim} Z$ holds in $r$.

Augmentation: Suppose $X \underset{\left(\alpha_{X}, \alpha_{Y}\right)}{\sim \longrightarrow} Y$ holds in $r$ and $V \subseteq W$.

$\forall t_{1}, t_{2} \in r, \tau\left(t_{1}[X W], t_{2}[X W]\right) \geq \alpha_{X W}$.

Clearly, $\tau\left(t_{1}[X], t_{2}[X]\right) \geq \alpha_{X}$

and $\tau\left(t_{1}[W], t_{2}[W]\right) \geq \alpha_{W}$.

Since $X \underset{\left(\alpha_{X}, \alpha_{Y}\right)}{\sim} Y$ holds in $r$, and by (7), we have $\exists t_{3} \in r$, such that

$$
\begin{aligned}
& \tau\left(t_{1}[X], t_{3}[X]\right) \geq \alpha_{X}, \\
& \tau\left(t_{1}[Y], t_{3}[Y]\right) \geq \alpha_{Y}, \\
& \tau\left(t_{2}[Z], t_{3}[Z]\right) \geq \alpha_{Z} .
\end{aligned}
$$

From (8), (9), (10) and (11) we have

$$
\tau\left(t_{1}[W], t_{3}[W]\right) \geq \alpha_{W}, \text { hence } \tau\left(t_{1}[X W], t_{3}[X W]\right) \geq \alpha_{X W} .
$$

Since $\tau\left(t_{1}[W], t_{3}[W]\right) \geq \alpha_{W}$ and $V \subseteq W$, then $\tau\left(t_{1}[V], t_{3}[V]\right) \geq \alpha_{V}$.

By (10), we obtain $\tau\left(t_{1}[Y V], t_{3}[Y V]\right) \geq \alpha_{Y V}$.

Since $U=R-X Y W \subseteq Z$ then $\tau\left(t_{2}[U], t_{3}[U]\right) \geq \alpha_{U}$.

From (12), (13) and (14), it follows that $X W \underset{\left(\alpha_{X W}, \alpha_{Y V}\right)}{\sim} Y V$ holds in $r$. 
Transitivity: Suppose $X \underset{\left(\alpha_{X}, \alpha_{Y}\right)}{\sim} Y$, and $Y \underset{\left(\alpha_{Y}, \alpha_{Z}\right)}{\sim} Z$ hold in $r$.

$$
\forall t_{1}, t_{2} \in r, \tau\left(t_{1}[X], t_{2}[X]\right) \geq \alpha_{X} .
$$

Since $X \underset{\left(\alpha_{X}, \alpha_{Y}\right)}{\longrightarrow} Y$ holds in $r$ then $\exists t_{3} \in r$ such that

$$
\begin{aligned}
& \tau\left(t_{1}[X Y], t_{3}[X Y]\right) \geq \alpha_{X Y}, \\
& \tau\left(t_{2}[X(R-Y)], t_{3}[X(R-Y)]\right) \geq \alpha_{X(R-Y)} .
\end{aligned}
$$

Since $Y \underset{\left(\alpha_{Y}, \alpha_{Z}\right)}{\longrightarrow} Z$ holds in $r$, then $\exists t_{4} \in r$, such that

$$
\begin{aligned}
& \tau\left(t_{1}[Y Z], t_{4}[Y Z]\right) \geq \alpha_{Y Z}, \\
& \tau\left(t_{3}[Y(R-Z)], t_{4}[Y(R-Z)]\right) \geq \alpha_{Y(R-Z)}
\end{aligned}
$$

First, we show that $\tau\left(t_{1}[X], t_{4}[X]\right) \geq \alpha_{X}$.

By (18), we have $\tau\left(t_{1}[X \cap Y Z], t_{4}[X \cap Y Z]\right) \geq \alpha_{X \cap Y Z}$.

Since $X-Y Z \subseteq R-Z$, and by (5), it follows that $\tau\left(t_{3}[X-Y Z], t_{4}[X-Y Z]\right) \geq \alpha_{X-Y Z}$.

By (17) and $X-Y Z \subseteq R-Z$ then $\tau\left(t_{2}[X-Y Z], t_{4}[X-Y Z]\right) \geq \alpha_{X-Y Z}$.

Therefore, $\tau\left(t_{1}[X-Y Z], t_{4}[X-Y Z]\right) \geq \alpha_{X-Y Z}$.

Combining (20) with (21), we obtain $\tau\left(t_{1}[X], t_{4}[X]\right) \geq \alpha_{X}$.

Next, we show that $\tau\left(t_{1}[Z-Y], t_{4}[Z-Y]\right) \geq \alpha_{Z-Y}$.

By (18), it is easy to see that $\tau\left(t_{1}[Z-Y], t_{4}[Z-Y]\right) \geq \alpha_{Z-Y}$.

Final, we show that $\tau\left(t_{2}[W], t_{4}[W]\right) \geq \alpha_{W}$, where $W=R-X(Z-Y)$.

Since $R-X Y Z \subseteq R-Z$, and $R-X Y Z \subseteq R-Y$, by (18) and (19),

we obtain $\tau\left(t_{2}[R-X Y Z], t_{4}[R-X Y Z]\right) \geq \alpha_{R-X Y Z}$.

From (16), (18) and (19), we have $\tau\left(t_{2}[Y], t_{4}[Y]\right) \geq \alpha_{Y}$.

Since $W \subseteq Y(R-X Y)$, by (22) and (23), it follows that $\tau\left(t_{2}[W], t_{4}[W]\right) \geq \alpha_{W}$.

Consequently, $X \underset{\left(\alpha_{X}, \alpha_{Z-Y}\right)}{\sim \longrightarrow} Z-Y$ holds in $r$.

The following rules are easily to obtained
FMVD4 (Reflexivity):
If $Y \subseteq X$ then $X \underset{\left(\alpha_{X}, \alpha_{Y}\right)}{\sim \sim} Y$

FMVD5 (Union):

If $X \underset{\left(\alpha_{X}, \alpha_{Y}\right)}{\sim \longrightarrow} Y$ and $X \underset{\left(\alpha_{X}, \alpha_{Z}\right)}{\sim \sim \sim} Z$ then $X \underset{\left(\alpha_{X}, \alpha_{Y Z}\right)}{\sim \longrightarrow} Y Z$

FMVD6 (Decomposition): If $X \underset{\left(\alpha_{X}, \alpha_{Y}\right)}{\sim} Y$ and $X \underset{\left(\alpha_{X}, \alpha_{Z}\right)}{\sim} Z$ then $X \underset{\left(\alpha_{X}, \alpha_{(Z-Y}\right)}{\sim}(Z-Y)$

$$
\text { and } X \underset{\left(\alpha_{X}, \alpha_{(Y-Z)}\right)}{\sim \longrightarrow}(Y-Z)
$$

FMVD7 (Pseudo-transitivity): If $X \underset{\left(\alpha_{X}, \alpha_{Y}\right)}{\sim \longrightarrow} Y$ and $Y W \underset{\left(\alpha_{Y W}, \alpha_{Z}\right)}{\sim \longrightarrow} Z$ then

$$
X W \underset{\left(\alpha_{X W}, \alpha_{(Z-Y W)}\right.}{\sim}(Z-Y W)
$$

Rules relate $(\alpha, \beta)$-ffds and $(\alpha, \beta)$-fmvds

$$
\begin{array}{ll}
\text { FFD-FMVD1 (Replication): } & \text { If } X \underset{\left(\alpha_{X}, \alpha_{Y}\right)}{\sim} Y \text { then } X \underset{\left(\alpha_{X}, \alpha_{Y}\right)}{\sim} Y \\
\text { FFD-FMVD2 (Coalescence): } & \text { If } X \underset{\left(\alpha_{X}, \alpha_{Z}\right)}{\sim} Z, Y \underset{\left(\alpha_{Y}, \alpha_{Z^{\prime}}\right)}{\sim} Z^{\prime} \text {, where } Z^{\prime} \subseteq Z, Y \cap Z=\emptyset \\
& \text { then } \underset{\left(\alpha_{X}, \alpha_{Z^{\prime}}\right)}{\sim} Z^{\prime}
\end{array}
$$

FFD-FMVD3 (Mixed pseudotransivity): If $X \underset{\left(\alpha_{X}, \alpha_{Y}\right)}{\sim} Y, X Y \underset{\left(\alpha_{X Y}, \alpha_{Z}\right)}{\sim} Z$ then $X \underset{\left(\alpha_{X}, \alpha_{(Z-Y}\right)}{\sim}(Z-Y)$

Definition 4.3. Let $F$ and $G$ be sets of $(\alpha, \beta)$-ffd and $(\alpha, \beta)$-fmvd on relation scheme $S=(R, \vec{s}, \vec{\alpha})$. The closure of $F \cup G$, denoted by $(F, G)^{+}$, is the set of all $(\alpha, \beta)$-ffds and $(\alpha, \beta)$-fmvds that can be derived from $F \cup G$ by repeated application of rules in the set \{FFD1-FFD6, MVD1-FMVD7, FFD-FMVD1-FFD-FMVD3\}.

Theorem 4.2. Let $S=(R, \vec{s}, \vec{\alpha})$ be a scheme relation, $X$ be a subset of $R$, then we can partition

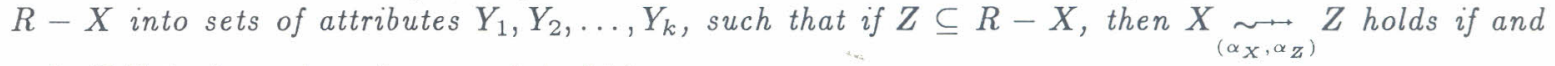
only if $Z$ is the union of some of the $Y_{i}$ 's. 
Proof. Similar to classical case, see the proof in [11].

We call the above sets $Y_{1}, Y_{2}, \ldots, Y_{k}$ constructed for $X$ from a set of $(\alpha, \beta)$-ffds and $(\alpha, \beta)$-fmvds $D$ the dependency basis for $X$ (with respect to $D$ ).

Theorem 4.3. Rules FFD1-FFD6, FMVD1-FMVD7, FFD-FMVD1-FFD-FNVD3 are complete on scheme $S=(R, \vec{s}, \vec{\alpha})$ when the following condition holds:

For each $A_{i} \in R$, there exists at least one pair of elements $a_{i}, b_{i} \in \operatorname{dom}\left(A_{i}\right)$ such that $s_{i}\left(a_{i}, b_{i}\right) \leq \alpha_{i}$.

Proof. Suppose $F, G$ are sets of $(\alpha, \beta)$-ffds and $(\alpha, \beta)$-fmvds on scheme $\mathcal{S}, d$ is a $(\alpha, \beta)$-ffd or $(\alpha, \beta)$ fmvd with left side is $X$ and $d$ does not follow from $F, G$ by axioms.

Let $\left\{Y_{1}, Y_{2}, \ldots, Y_{k}\right\}$ be a dependency basics for $X$ respect to $F \cup G$.

We set $X^{*}=\left\{A \in R \mid X \underset{\left(\alpha_{X}, \alpha_{A}\right)}{\sim} A \in(F, G)^{+}\right\}$.

Since $X \underset{\left(\alpha_{X}, \alpha_{X^{*}}\right)}{\sim} X^{*} \in(F, G)^{+}$then $X \underset{\left(\alpha_{X}, \alpha_{X^{*}}\right)}{\sim} X^{*} \in(F, G)^{+}$. Hence $X \underset{\left(\alpha_{X}, \alpha_{R-X^{*}}\right)}{\sim \longrightarrow} R-X^{*} \in$ $(F, G)^{+}$. By Theorem 4.2, we have $R-X^{*}=\bigcup_{i=1}^{m} W_{i}$, where $W_{i} \in\left\{Y_{1}, Y_{2}, \ldots, Y_{k}\right\}$.

We now construct the following relation $r$ on scheme $S$

\begin{tabular}{ccccc}
$X^{*}$ & $W_{1}$ & $W_{2}$ & $\ldots$ & $W_{m}$ \\
\hline$\left(a_{i}\right)_{i \in I_{0}}$ & $\left(a_{i}\right)_{i \in I_{1}}$ & $\left(a_{i}\right)_{i \in I_{2}}$ & $\ldots$ & $\left(a_{i}\right)_{i \in I_{m}}$ \\
$\left(a_{i}\right)_{i \in I_{0}}$ & $\left(b_{i}\right)_{i \in I_{1}}$ & $\left(a_{i}\right)_{i \in I_{2}}$ & $\ldots$ & $\left(a_{i}\right)_{i \in I_{m}}$ \\
$\left(a_{i}\right)_{i \in I_{0}}\left(a_{i}\right)_{i \in I_{1}}$ & $\left(b_{i}\right)_{i \in I_{2}}$ & $\ldots$ & $\left(a_{i}\right)_{i \in I_{m}}$ \\
$\left(a_{i}\right)_{i \in I_{0}}$ & $\left(b_{i}\right)_{i \in I_{1}}$ & $\left(b_{i}\right)_{i \in I_{2}}$ & $\ldots$ & $\left(a_{i}\right)_{i \in I_{m}}$ \\
$\vdots$ & $\vdots$ & $\vdots$ & $\vdots$ & $\vdots$ \\
$\left(a_{i}\right)_{i \in I_{0}}$ & $\left(b_{i}\right)_{i \in I_{1}}$ & $\left(b_{i}\right)_{i \in I_{2}}$ & $\ldots$ & $\left(b_{i}\right)_{i \in I_{m}}$
\end{tabular}

where $X^{*}=\left(A_{i}\right)_{i \in I_{0}}, W_{j}=\left(A_{i}\right)_{i \in I_{j}}, I_{j} \subseteq\{1,2, \ldots, m\}, j=0, \ldots, m$.

First we show that $r$ satisfies $F$ and $G$.

Suppose $U \underset{\left(\alpha_{U}, \alpha_{V}\right)}{\sim} V \in F$. We set $W=\bigcup_{i \in I} W_{i}$, where $I=\left\{i: W_{i} \cap U \neq \emptyset, 1 \leq i \leq n\right\}$.

Clearly $U \subseteq X^{*} W$ then $X^{*} W \underset{\left(\alpha_{X^{*} W}, \alpha_{V}\right)}{\sim} V \in(F, G)^{+}$.

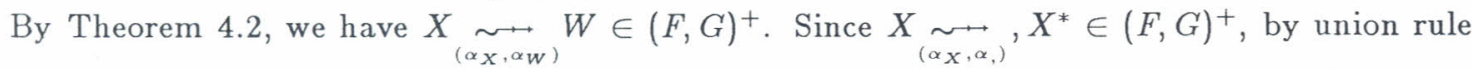

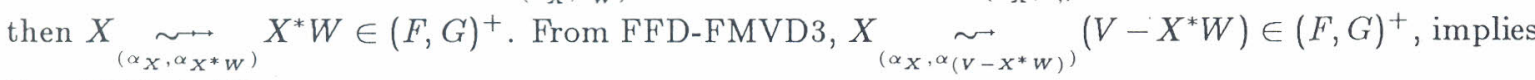
$V-X^{*} W \subseteq X^{*}$.

Let $t_{1}$ and $t_{2}$ be tuples of $r$ such that $\tau\left(t_{1}[U], t_{2}[U]\right) \geq \alpha_{U}$. By construction of $r$, it follows that $t_{1}[U]=t_{2}[U], t_{1}[W]=t_{2}[W], t_{1}\left[X^{*}\right]=t_{2}\left[X^{*}\right]$ so $\tau\left(t_{1}\left[X^{*} W\right], t_{2}\left[X^{*} W\right]\right) \geq \alpha_{X^{*} W}$. Hence $\tau\left(t_{1}[V], t_{2}[V]\right) \geq \alpha_{V}$. Therefore, $r$ satisfies $(\alpha, \beta)$-ffd $U \underset{\left(\alpha_{U}, \alpha_{V}\right)}{\sim} V$.

Now assume that $U \underset{\left(\alpha_{U}, \alpha_{V}\right)}{\longrightarrow} V \in G$. Since $U \subseteq X^{*} W$, by FMVD2 then $X^{*} W \underset{\left(\alpha_{X^{*} W}, \alpha_{V}\right)}{\longrightarrow} V \in(F, G)^{+}$.

We have $X \underset{\left(\alpha_{X}, \alpha_{X^{*} W}\right)}{\sim \rightarrow} X^{*} W \in(F, G)^{+}$, by FMVD3 then $X_{\left(\alpha_{X}, \alpha_{\left(V-X^{*} W\right)}\right.}^{\sim \cdots}\left(V-X^{*} W\right) \in(F, G)^{+}$.

By Theorem 4.2, $V-X^{*} W=\bigcup_{i \in I_{1}} W_{i}, I_{1} \subseteq\{1, \ldots, m\}$. For any pair tuples $t_{1}, t_{2} \in r$, $\tau\left(t_{1}[U], t_{2}[U]\right) \geq \alpha_{U}$, by construction of $r, t_{1}[U]=t_{2}[U]$. There exists a tuple $t_{3}$ which is defined by $t_{3}\left[V-X^{*} W\right]=t_{1}\left[V-X^{*} W\right]$, and $t_{3}\left[R-\left(V-X^{*} W\right)\right]=t_{2}\left[R-\left(V-X^{*} W\right)\right]$.

It is easy to see that $t_{3}$ is a tuple in $r$ and $t_{3}[U]=t_{1}[U], t_{3}[V]=t_{1}[V]$ (because $t_{1}[W]=t_{3}[W]$ ), $t_{3}[R-U V]=t_{2}[R-U V]$.

Hence $\tau\left(t_{1}[U], t_{3}[U]\right) \geq \alpha_{U}, \tau\left(t_{1}[V], t_{3}[V]\right) \geq \alpha_{V}, \tau\left(t_{2}[R-U V], t_{3}[R-U V]\right) \geq \alpha_{R-U V}$.

Therefore, $r$ satisfies $(\alpha, \beta)$-fmvd $U \underset{\left(\alpha_{U}, \alpha_{V}\right)}{\sim} V$.

We now show that $d$ does not hold by $r$.

Suppose that $d=X \underset{\left(\alpha_{X}, \alpha_{Y}\right)}{\sim} Y$. Since $d \notin(F, G)^{+}$then $Y \nsubseteq X^{*}$. 
By construction of $r$, there exist two tuples $t_{1}, t_{2} \in r$, such that $t_{1}[Y] \neq t_{2}[Y]$. Furthermore, we have $\tau\left(t_{1}[Y], t_{2}[Y]\right) \geq \alpha_{Y}$. But $t_{1}[X]=t_{2}[X]$ then $\tau\left(t_{1}[X], t_{2}[X]\right) \geq \alpha_{X}$. Hence, $r$ does not hold $X \underset{\left(\alpha_{X}, \alpha_{Y}\right)}{\sim} Y$.

Now assume that $d=X \underset{\left(\alpha_{X}, \alpha_{Y}\right)}{\sim \longrightarrow} Y \notin(F, G)^{+}$, and $d$ holds on $r$.

By construction of $r$, it is easy to show that $Y=X^{\prime} \cup W^{\prime}$, where $X^{\prime} \subseteq X^{*}, W^{\prime}=\bigcup_{j \in J} W_{j}$, $J \subseteq\{1, \ldots, m\}$.

We have $X \underset{\left(\alpha_{X}, \alpha_{X^{\prime}}\right)}{\longrightarrow} X^{\prime} \in(F, G)^{+}$, and by Theorem 4.2, $\underset{\left(\alpha_{X}, \alpha_{W^{\prime}}\right)}{\sim} W^{\prime} \in(F, G)^{+}$. From union rule, we have $X \underset{\left(\alpha_{X}, \alpha_{Y}\right)}{\sim} Y \in(F, G)^{+}$, contradition. Thus $d$ does not hold on $r$. The proof is complete.

\section{CONCLUSION}

This paper deals with fuzzy data dependencies in fuzzy relational databases. We give the definitions of fuzzy functional and multivalued dependencies. Furthermore, we discuss the inference rules of these dependencies. The soundness and completeness are proved. A futher study involving the definitions of fuzzy join dependency, normal forms for the fuzzy relational databases has been on going.

\section{REFERENCES}

[1] Bhattacharjee T. K., Mazumdar A. K., Axiomatisation of fuzzy multivalued dependencies in a fuzzy relational data model, Fuzzy Sets and Systems 96 (1998) 343-352.

[2] Buckles B.P., Petry F.E., Information-theoretic characterization of fuzzy relational databases, IEEE Trans. Syst. Man Cybernet 13 (1983) 74-77.

[3] Chen G., Kerre E. E., Vandenbulcke J., A computational algorithm for the FFD transitive closure and a complete axiomatization of fuzzy functional dependence, International Journal of Intelligent Systems 9 (1994) 421-439.

[4] Cubero J. C., Vila M.A., A new definition of fuzzy functional dependency in fuzzy relational databases, International Journal of Intelligent Systems 9 (1994) 441-448.

[5] Dubois D., Prade H., Possibility Theory : An Approach to Computerized Processing of Uncer-. tainty, Plenum Press, New York, 1988.

[6] Jyothi S., Babu M.S., Multivalued dependencies in fuzzy relational databases and lossless join decomposition, Fuzzy Sets and Systems 88 (1997) 315-332.

[7] Kraft D.H., Petry F.E., Fuzzy information systems: managing uncertainly in databases and information retrieval systems, Fuzzy Sets and Systems 90 (1997) 183-191.

[8] Petry F., Bosc P., Fuzzy Databases : Principles and Applications, Kluwer, Norwell, MA, 1996.

[9] S. Shenoi, A. Melton, An extended version of the fuzzy relational database model, Information Sciences 52 (1990) 35-52.

[10] S. Shenoi, A. Melton, and L. T. Fan, Functional dependencies and normal forms in the fuzzy relational database model, Information Sciences 60 (1992) 1-28.

[11] Ullman J. D., Principles of Database system, Comp. Science Press, 1980.

[12] L. T. Vuong, H. Thuan, A relational databases extended by application of fuzzy set theory and linguistic variables, Computers and Artifical Intelligence 8 (2) (1989) 153-168.

[13] Yazici M. et al., The integrity constrains for similarity - based fuzzy relational databases, International Journal of Intelligent Systems 13 (1998) 641-659.

Ho Thuan - Institute of Information Technology.

Received August 10, 2000

Tran Thien Thanh - Pedagogical Institute of Quy Nhon. 